\title{
Chapter 8 \\ Closing Thoughts, Insights and Resources for Digital Touch Communication Research and Design
}

\begin{abstract}
This chapter closes the book with a note on thematic directions, in response to the speculative and emergent character of digital touch communication, signalling our desire and need to keep the conversation open. We point to the significance of a social take on digital touch, particularly with reference to the types of questions this perspective raises and the way it positions technology in relation to people and society more generally. We draw attention to the research insights on digital touch communication discussed throughout the book that may inform design. Finally, we comment on the theoretical and methodological routes that we have taken to research digital touch communication, and draw on the ideas and research presented in this book to sketch an emergent research and design framework for digital touch communication.
\end{abstract}

\subsection{A Social Perspective on Digital Touch}

The social take on digital touch provided in this book is significant for what gets brought into the scope of research and design, the types of questions raised, and the ways that technology is positioned as intrinsically linked to social relations, mutually shaping each other as they are developed and maintained.

Throughout the book, we have illustrated how developments in sensory digital technologies are bringing touch to the fore in ways that move digital communication beyond 'ways of seeing' to include new 'ways of feeling' and the competing discourses of desire and anxiety that this gives rise to. We have shown that this shift requires us to take new measure of digitally mediated touch, or 'digital touch', as a communicational resource. Through extensive engagement with the research literature and state-of-the-art digital touch devices, alongside a range of illustrative case study examples, we have explored what digital touch is (currently) and what it may come to be, how it is designed and imagined, and discussed people's imaginations of and responses to its communicative potentials and limitations. We examined how touch is conceptualized, imagined and experienced by people through different 
technologies and in different interactional contexts, the aspects of digital touch that are central to a range of communicational situations, how people improvise around digital touch and the skills, experiences and communicative repertoires that they draw on to do so. We have examined how designers and users take up the resources of touch that are available to them, and how the sensory-affective qualities and affordances, and the materiality of different touch technologies feature in different social and situated contexts. Through a social perspective, we have sought to understand how people take up and use digital touch technologies to mimic or supplement existing touch capacities and practices, heighten touch experiences, extend touch newly - for example, across distance, to be stored and shareable, or to reconfigure touch digitally to reshape what counts as touch and who and how we touch in various contexts. In doing so, the book documents key social resources for touch, the touch interactions supported and the kinds of touch communication practices that are being designed and identified, the social potentials and constraints of touch that are taken up by the designers of 'digital touch'. This extends to how digital touch technologies are situated and embedded in the wider contexts and experiences of everyday life, and how touch technologies require people to reimagine these for the future. Technological development in this area is still somewhat in its infancy and often remains at a 'proof of concept' stage; nonetheless, it is bringing a diverse set of techniques and engineering capacities, as well as various approaches to informing or underpinning designs and applications, depending on the area of use.

\subsection{Insights for Digital Touch Communication}

This book makes the case for a socially orientated and interdisciplinary approach to digital touch communication research and points to insights on key touch resources, dimensions and considerations that provide an emergent agenda for further digital touch communication research and design - starting routes or jumping off points, from which to further develop digital touch communication.

\subsubsection{Social Norms and Digital Touch}

Attending to the social norms that underpin people's touch interaction and communication, and how these are negotiated in social encounters, can provide a starting point from which to leverage understanding of the sociality of the tactile regime (Cranny-Francis 2011) in which they are embedded. Social norms of touch developed in relation to 'direct' touch, and its associated etiquettes and practices, have been (and will be) brought into the use and design of digital touch devices, systems and environments, albeit in uneven ways. Like digitally mediated visual communication, some norms and practices will be disrupted in 'translation', and it is likely that some new touch capacities and interactions will be elicited. In this fluid mix, 
unintended and unexpected consequences for how we communicate with others via touch will emerge. We suggest that this points to a need to consistently engage with the social and move beyond an emphasis on design explorations and point to solutions. Touch norms are significant in that they provide insights into the shared usage of touch for making culturally shared meaning of touch, and expectations of touch, which supports the imagination and design of digital touch communication. Understanding and reflecting on our own touch norms, as well as those of the people we research and/or design for, is therefore a useful route to recognising and benefiting from the potentials for difference and cultural flexibility towards new possibilities for designing digital touch communication. While on the one hand, understanding touch within the cultural complexities of the contemporary communicational landscape, characterised as it is by super-diversity, challenges the concept of social norms as stable and universal; on the other, gendered and cultural norms persist, perhaps more than ever given the hegemonic effect of the global circulation of technology. Given that social norms of touch are designed into and realised through the affordances of digital technologies, an awareness of the social norms of touch and how these regulate touch practices can help us to question, and/or engage newly with touch, from the mundane vibration of a phone in our pocket, to robotic-touch, and the innovation of contactless touch: the who, what, where, how and when of digital touch.

\subsubsection{Touch Connections}

The concepts of presence, absence and connection are significant technologically, socially, communicatively, sensorially, emotionally and imaginatively and, as such, central to the design and use of digital touch for communication. A social perspective on connection drives home the complexity of social presence and asserts that it goes well beyond being physically co-located. This opens up the design space and scope of what we might mean by producing 'presence' and 'connection' through digital touch, and suggests the need to attend to the situated social and sensorial meanings that emerge through interaction moments of which digital touch is a part. It also brings a number of tensions to the surface that can serve as important considerations for design, including the question of mimicking or replicating human touch versus touch at a symbolic or imagined level, which may give rise to new forms of sharing or experiencing through touch. Related to this is the tension between the significance of specific touch interfaces - their materiality, sensorial affordances, social connotations and functionality - and the idea that these might move into the background, or be personalised, and function as 'mere' mediators or enablers of digital touch communication. Throughout this book, we have argued that interfaces can be transformative or reductionist, depending on how advanced or situationally appropriate they 'feel'. Building on this, we suggest that they are strengthened by being sensitive to differently situated and experiencing bodies - shaped through the intersections of age, gender, different abilities, race and culture and personal 
preferences. We have also discussed how new touch technologies will emerge into an existing polymedia environment or technoscape, rather than existing in isolation, leading to notions of ambient touching and tactile presence with touch a part of the broader digital sensory terrain.

\subsubsection{Sociotechnical Imaginaries of Digital Touch}

This book has explored and made legible emerging sociotechnical imaginaries of digital touch to address how touch practices might be shaped through the uses of technology, and how this might in turn shape notions and practices of communication. We have fleshed out the sociality of digital touch communication by making legible emergent imaginaries of digital touch communication, providing critical understanding and insight on digital touch communication futures, and excavating and interrogating the features of sociotechnical imaginaries that 'tacitly' constrain and afford research and design of digital touch. We have discussed research participants' sociotechnical imaginaries of digital touch communication related to the body, temporality and spatiality, and drawn out three key themes that emerged through these articulations and deployments of the sociotechnical imaginary. These include speculations on touch with regard to the politics of touch, the representation of touch and the ethics of touch.

In addition to understanding the sociotechnical imaginaries that circulate among the users and contexts that we are researching and designing for, we make the case for exploring our own sociotechnical imaginaries, towards an explicit awareness of how they underpin and drive our research and design of digital touch. Such an awareness can, we argue, enable us to better articulate the social parameters that underpin our work, in order to understand how our imaginaries 'tacitly' constrain and afford research and design and provide a springboard from which to move beyond, extend, or disrupt them. The sociotechnical imagination enables us to glimpse some aspects of potential digital touch futures, and to engage with thinking what we want from the sociality of digital touch communication. Exploring sociotechnical imaginaries is therefore a vital resource towards a future methodology and agenda for the relatively uncharted territory of digital touch.

\subsubsection{The Ethics of Touch}

Across the book, we have raised key questions of touch ethics and values in digitally mediated contexts. We brought the complexities of 'the body' in its physiological, socio-cultural and sensory manifestations to the forefront of digital touch, with a view to both exploring existing moments of digital touch interaction and designing new ones. Key sensitivities included the kinds of touches, movements, mobilities and socialities inscribed in touch interfaces and wider user experiences, and how we 
imagine and understand bodies, in terms of their agency, ability and diversity. We argue that one way of embedding ethical values in the design of touch technologies is to attend to the sociotechnical imaginaries that guide our research and design, and the imaginations of those we design with and for, as well as the relationships and environments we create, that is, what kinds of relationships are enabled or restricted, whose rights are upheld, who is empowered, or not.

Just as we have proposed attending to touch in its different digital-material manifestations and its implications for bodies and social relationships, we have also discussed key ethical concepts, such as consent, trust and control, as dynamic and multifaceted. We highlighted a tension between liberating and censoring digital touch, which we have not fully resolved. Instead, we suggest more research is needed to understand the social and psychological implications of emerging touch technologies, not just after the fact but also, crucially, at those opportune moments when early concepts, prototypes, user scenarios and wider discourses allow us to access social and sensory meanings and connotations of significance for future designs. This involves actively engaging with touch boundaries - not just as sets of rules but as talking points and sensitivities. Our research suggests that digital mediation has the potential to change sensations, communication practices and social and relations but that existing social boundaries still exist; they are felt and negotiated, both in the immediate interaction context and in wider meanings and connotation of mediated touch. Bringing the sensitivities and complexities of touch to the forefront of design - and making them a talking point in and through design - is, we suggest, one step towards safeguarding ethical touch.

\subsection{Methodologies for Digital Touch}

In this book, we have discussed the many methodological challenges of researching digital touch communication at a time when technologies are evolving rapidly and are not yet 'domesticated', and methods and theories remain under-developed. Including the challenge of researching digital touch technologies that are unstable, lab-bound, researching digital touch with under-developed methods and theories; and the difficulty in observing, interpreting and making 'felt' touch experiences.

In response to these challenges, we have made the case for attending to the multimodal and multi-sensorial aspects of touch, making the sociality and sensorality of digital touch our starting point and focus. As such, we argue for an approach to digitally mediated touch as a communicative mode (a set of resources and principles for their organization and use), a sensorial experience entangled in the materiality and sociality of the body, the environment and technologies. Alongside this, we have maintained the significance of interdisciplinary dialogues for understanding touch, in particular with art, neuroscience, $\mathrm{HCI}$ and computer science, engineering, and design. In our broad theoretical framing, we have used a range of methods to engage participants in creative processes, making and bodily touchy-activities with 
themselves, others, materials and objects, and to deliberately go beyond the linguistic and the individual. These methods have included the development of design briefs, the design and use of technology-probes and artistic provocations, ethnographic encounters, in-depth interviews and focus groups centred around demonstrations with digital devices and environments, prototyping, speculative scenarios and role play with touch devices, and video re-enactments and walk-throughs (in the home, gallery, and virtual environments). In particular, we have illustrated the potential of prototyping to bridge interdisciplinary differences in the context of social science research collaborations with other disciplines, in order to gain access to and generate digital touch experiences and imaginations for research purposes. Collectively these methods provide opportunities for reflection on the rich complexities of touch and have proven to be particularly adept at accessing participants' sociotechnical imaginaries of digital touch communication. Generating new research spaces for digital touch can help to open up new routes for participants to reimagine touch and to explore the new social boundaries of digital touch communication. It has also enabled us to access participants' sociotechnical imaginaries of digital touch communication and to both explore and re-orientate to its past, present and futures.

\subsection{An Emergent Research and Design Framework for Digital Touch Communication}

Designing digital touch is complex, and we have set out to explore ways to prompt and support a broad, nuanced conception of digital touch. For example, drawing our research on digital touch communication into the development of a card-based resource, the Designing Digital Touch Toolkit, to support engagement with the complexities of working with touch across different stages of Design Thinking. We suggest that social science research can help to expand design processes through the emphasis that it puts on the social, sensory and communicative properties of touch to encourage greater awareness, discussion and investigation of digital touch. In this way, social science can raise questions to help designers reflect newly on their own and others' touch experiences; offer more conceptual or abstract prompts and provocation for thought or action; and suggest structured exercises to work through specific design elements for digital touch communication.

Building on the broad social, multimodal and sensorial foundation that underpins our work, and drawing on the ideas and research presented across the book chapters, we close this book by sketching an emergent framework to inform and support social, sensory and ethical research and design of digital touch communication (experiences, devices, systems and environments). This provisional Digital Touch Communication framework (Fig. 8.1) provides socially oriented frames of attention and an initial set of investigatory dimensions emerging out of the work discussed in this book with which to think through the social and sensorial 


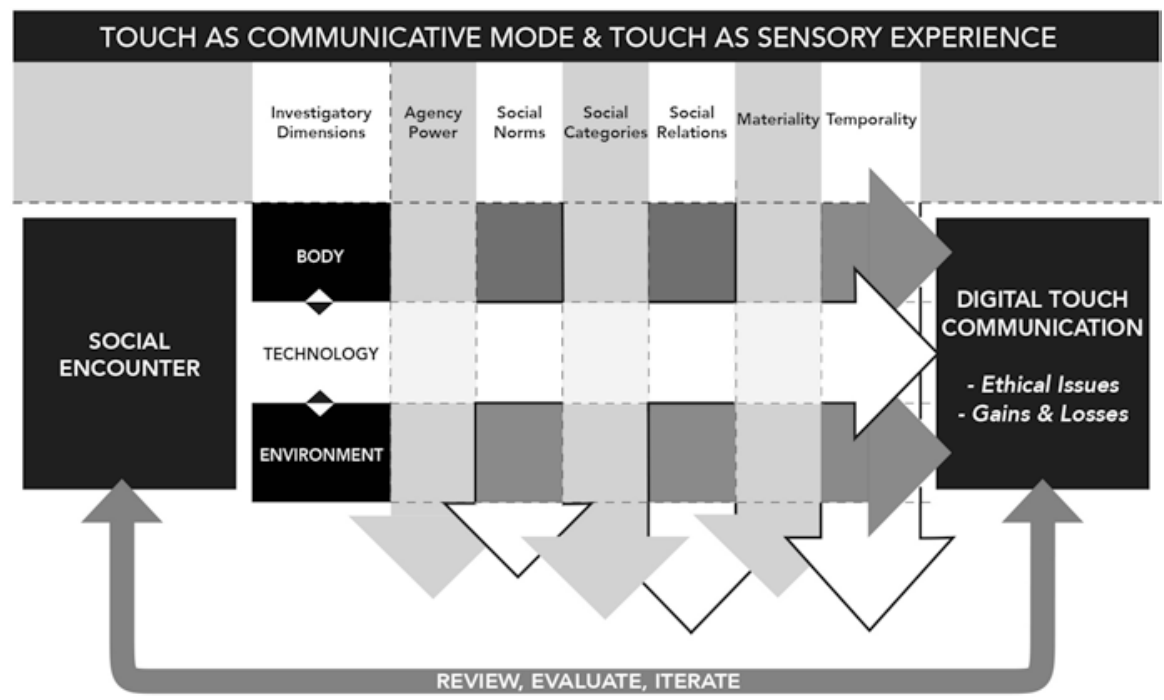

Fig. 8.1 Initial InTouch Research and Design Framework for Digital Touch Communication

components of digital touch communication. These are designed to support researchers and/or designers in the critical design and analysis of digital touch communication by attending to, and reflecting on, the ethical considerations raised, and the communicative gains and losses realised when touch is digitally mediated. At this early stage, the framework is tentative and intended as a springboard for future development and conceptualisation.

The over-arching 'frame of attention' that structures the framework, built on our theoretical stance, articulates a simultaneous concern with touch as a communicative mode and as sensorial experience. It requires, on the one hand, analytical attention towards the different modes and modal resources that are available within a given digital touch communication encounter (e.g. of movement, posture and gesture, gaze, visual representational modes, or sound - speech or music), and how these are taken up by users and orchestrated in relation to touch communication. On the other hand, and closely related to this multimodal attention, the frame also accounts for, and encourages reflection on, the ways in which touch is part of multisensorial experience and meaning-making.

This framing draws attention to a set of inter-related initial investigatory dimensions, which are realised through (and actualise) the social orchestration of modes and sensorial experience: agency and power - who or what touches; social norms of touch; social categories related to touch (e.g. gender) (as discussed in other chapters, such categories are continuously constructed and differently meaningful, rather than deterministic); social relations through touch; materialities of touch; and tactile temporalities. These dimensions will be refined and added to through the findings of future case studies and the analytical work of InTouch. 




Fig. 8.2 An indication of the kinds of considerations and resources that might populate the Framework for Digital Touch Communication

We understand these social and sensorial dimensions of touch as being entangled in and produced through situated encounters (shaped by and shaping the social), in which the materiality and sociality of the body, the environment and technologies are key: these three interconnecting concepts (body, technology and environment) provide a second parallel set of investigatory considerations that structure the framework. While these three concepts are always in play and in interrelation, the framework can be used to bring them in and out of focus in order to emphasize specific aspects of digital touch within an encounter for the purposes of research and/ or design.

A digital touch communication encounter may refer to the design process itself and a user may include designers and/or researchers. Touch communication and sensorial experiences are understood as a part of the production (and result of) social encounters - between humans, humans and objects, or humans and robots - in social, sensory and material environments. These encounters (e.g. medical, professional, or personal relationships) are shaped through (and shape) larger social framings imbued with particular and varying touch histories and practices.

We have populated the diagram of the Framework below (Fig. 8.2) to indicate the kinds of considerations and resources that might be interrogated in relation to its investigatory dimensions. These are intended as illustrative rather than exhaustive and serve to give a sense of the communicative and sensorial aspects of digital touch communication that can be brought into view through the framework. The Digital Touch Communication Framework can also be used alongside other research and design tools or frameworks (e.g. the Double Diamond Design Framework) (Design Council 2007). 
The framework can be used in the process of designing a digital touch communication experience, or a specific digital touch device, system, or environment. It offers a variety of entry points into all design stages (i.e. discover, define, develop and deliver) as well as the iterative process of design evaluation and refinement. For instance, a designer can use the framework to bring a specific research or design consideration (e.g. Body and Agency) into focus, in relation to a specific user group and social encounter. They may use the framework to explore a particular dimension of touch, for instance they may interrogate how their design is or could utilise different tactile temporalities to consider the different impacts of these on their designed touch communication experience across the dimensions of the body, touch technology, and environment. The framework can be used to explore the design of a digital touch experience in a holistic way, looking across its dimensions by attending to how tweaks and changes in each 'cell' of the framework might shape that experience.

Similarly, in the context of researching digital touch communication, the framework provides a series of considerations to guide and frame attention. These may be used to generate interview or focus group questions, offer analytical dimensions in relation to specific digital touch communication devices as they feature in a particular social encounter, or provide a way to focus in on the body, technology, environment, temporality or other key aspects of the digital touch communication experience to sensitise the research to the multimodal, sensorial and social aspects of digital touch.

The thematic directions, emergent ideas, and provisional framework that this closing chapter, and the book more generally, offers highlights both the speculative and emergent character of digital touch communication and the value of bringing a social, multimodal and sensorial, perspective to the ongoing discussion of what people imagine and desire for digital touch communication, what it is and may come to be in our futures.

\section{References}

Cranny-Francis A (2011) Semefulness: a social semiotics of touch. Social Semiotics 21(4):463-481

Design Council (2007) Eleven lessons: managing design in eleven global companies. Design Council, London

Open Access This chapter is licensed under the terms of the Creative Commons Attribution 4.0 International License (http://creativecommons.org/licenses/by/4.0/), which permits use, sharing, adaptation, distribution and reproduction in any medium or format, as long as you give appropriate credit to the original author(s) and the source, provide a link to the Creative Commons licence and indicate if changes were made.

The images or other third party material in this chapter are included in the chapter's Creative Commons licence, unless indicated otherwise in a credit line to the material. If material is not included in the chapter's Creative Commons licence and your intended use is not permitted by statutory regulation or exceeds the permitted use, you will need to obtain permission directly from the copyright holder.

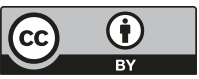

